\title{
Design of Fixed-Wing and Multi-Copter Hybrid Drone System for Human Body Temperature Measurement during COVID-19 Pandemic
}

\author{
ZAYED ALMHEIRI \\ Department of Mechanical \\ Engineering \\ Abu Dhabi University \\ Abu Dhabi, P.O. Box 59911 \\ UNITED ARAB EMIRATES
}

\author{
RAWAN ALEID \\ Department of Mechanical \\ Engineering \\ Abu Dhabi University \\ Abu Dhabi, P.O. Box 59911 \\ UNITED ARAB EMIRATES
}

\author{
SHARUL SHAM DOL \\ Department of Mechanical \\ Engineering \\ Abu Dhabi University \\ Abu Dhabi, P.O. Box 59911 \\ UNITED ARAB EMIRATES
}

\begin{abstract}
The purpose of this research is to conduct aerodynamics study and design a hybrid drone system of fixed-wing and multi-copter. The mission of this drone is to measure human body temperature during COVID19 pandemic. The specific aim of the drone is to fly and cover larger industrial areas roughly about $50 \mathrm{~km}^{2}$ with longer flying time than the conventional drone, of about 1.5 hours. The applications of the simulation software such as XFLR5 and ANSYS have a big impact in identifying areas that need to be improved for the drone system. XFLR5 software was used to compare the characteristics of different airfoils with highest lift over drag, $L / D$ ratio. Based on the airfoil selection, it was found that NACA 4412 airfoil produces the highest $L / D$ ratio. The detailed geometry of the drone system includes a fuselage length of 1.9 meters and wingspan of 2 meters. Moreover, 10 sheets of solar panels were placed along the wing for sustainable flight operation to cover wider areas of mission. The structural analysis was done on ANSYS to test the elastic stress, equivalent strain, deformation, factor of safety pressure as well as lift and drag forces under various operational conditions and payloads. The landing gear was analyzed for harsh landing. ANSYS Computational Fluid Dynamics (CFD) was utilized to study the aerodynamics of the drone at different parameters such as the velocities and angles of attack during the operation. This design ensures the stability of the drone during the temperature measurement phase. The best thermal-imaging camera for such purpose would be the Vue Pro R 336, $45^{\circ}$ radiometric drone thermal camera with a resolution of $640 \times 512$ pixels. This camera has the advantage of a permanent continuous out focus that give the ability of taking measurements even if there was changing on the altitude or any kind of vibrations.
\end{abstract}

Key-Words: - Aerodynamics; CFD; COVID-19; Drag; Drone; Fixed-wing; Lift; Multi-copter; Thermal camera

Received: September 26, 2020. Revised: January 23, 2021. Accepted: March 1, 2021. Published: March 16, 2021.

\section{Introduction}

This paper aims to discuss various stages of design process of a hybrid drone system, and ways to enhance of certain parameters such as aerodynamics stability and longer flying time to satisfy mission requirement. The objective of the research was to design an aerodynamic efficient hybrid drone system of fixed-wing and multi-copter combination. Using a high-end thermal camera embedded with the drone, the purpose was to scan human body temperatures over a wider space such as in industrial areas during this COVID-19 pandemic period. The application of such drone will be crucial to identify suspected cases faster than the stationary cameras used currently at the selected checkpoints.

The payload considered in the design is the 90-g thermal camera with the total gross weight of the drone is $6.7 \mathrm{~kg}$. For the thermal camera to provide high accuracy temperature reading, the drone should fly at the altitude of between $3-3.5 \mathrm{~m}$ [1]. The specific aim of the drone is to fly and cover certain industrial areas in Abu Dhabi, United Arab Emirates (Fig. 1) roughly about $50 \mathrm{~km}^{2}$ over the 1.5 hours flying mission time. The transition from one area to another will be done by the fixed-wing function to reduce power consumption [2-3]. This allows the drone to take advantages of both the characteristics of fixed-wing, like high-speed and wider range, and 
of multi-copter, such as hovering, high manoeuvrability, vertical take-off and landing.

Currently, drones have proved capabilities in achieving missions in various fields such as: search and rescue (SAR) as shown in Figure 2, agriculture, patrolling, and transport [1, 4-6]. Drone's field is growing and enhancements are done, which resulted in improved the capabilities over time, and fulfilled ever-changing mission requirement with safer and lighter drone due to the advanced material, lighter rotors and better aerodynamics performance [7-9. Drones are excellent for aerial photos, video, and imagery sets. For instance, precision farming uses drones for a variety of agricultural needs, such as fertilizer and insecticide spraying, weeds identification, and crop health monitoring. In conjunction with the relatively low costs of many models, a broad number of operators have access to drones. Precision point of view, the precise programming and operation of unmanned aerial vehicles to correct positions is based on GPS (Global Positioning System). Drones may also collect useful data for defenses and recovery operations during and after natural disasters.

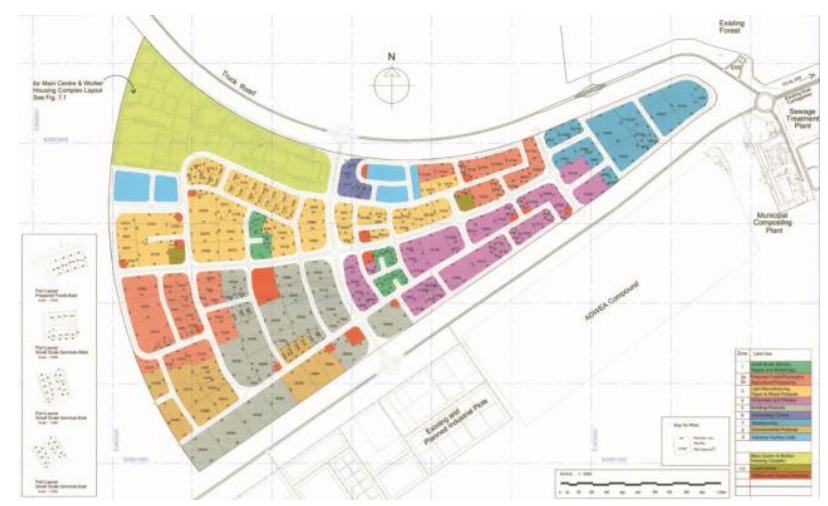

Figure 1: Al Ain UAE industrial area

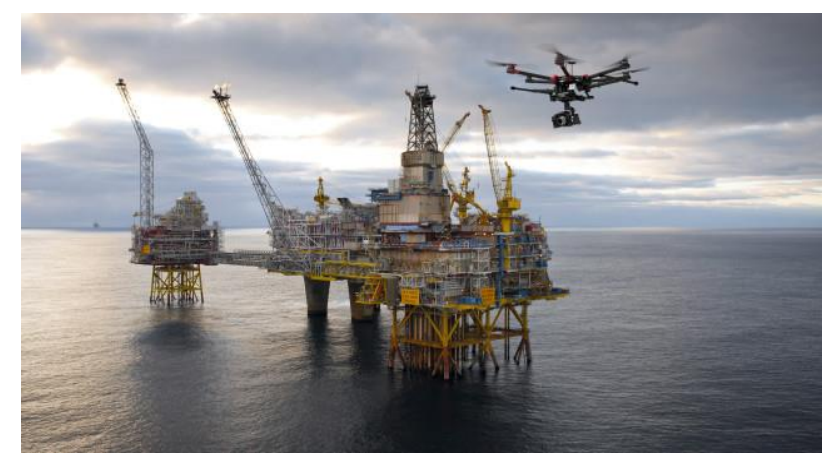

Figure 2: Offshore oilrig SAR using drone [6]

Although there are many advantages to use drones, their deployment is also met with a range of perceived challenges [10]. These concerns should be considered, especially since drones can be used under various circumstances. Like, the safety and privacy as if people link their social media accounts directly to the drone's controlling systems, all information becomes vulnerable, which enable unauthorized third parties to sensitive files. Also, in terms of spying when criminal elements use drones to achieve their criminal intent, they are now vulnerable to violation of the privacy of their targeted victims. Drones record their daily routine and track suspected activities without the permission of an individual, group or authority to be the object of interest risk of atrocities. In the past, there are many drones fired arsenals mistakenly to civilians that have caused considerable casualties, injuries and damage to a given area.

Moreover, drones aerodynamics performances have improved resulting in extended missions and higher degrees of automated flight. Drones are divided into two primary sectors: multirotor systems, which usually have 4 to 8 propellers that allows vertical take-off and landing (VTOL). Also, they can achieve better manoeuvrability; however, the propellers create enormous drag force, which results in reducing its performance. The other sector is the fixed-wing drones that require a runway and not capable of a vertical take-off. Nevertheless, fixed-wing drones can carry a heavier payload compared to multi-rotors. In missions that require longer range, high speed, and heavy load, the fixed wing drone is more preferred.

Furthermore, it is known that the fixed-wing drone has longer range but it comes with difficult landing since it needs a runway, especially in crowded cities. On the other hand, the multi-copter can achieve missions in mountain areas without the need of a runway. The choice of the drone depends on the task the drone must achieve. For example, if long endurance is required then the choice is fixedwing, but if high maneuverability is required, the choice will be multi-copter. Hybrid drone allows the features of both fixed-wing and multi-copter to be optimized. Hybrid drones are put into different categories: tilt rotor, tilt wing, rotor-wing. The tilt rotor allows movements of the rotors around their arm axes to switch between VTOL and horizontal flying. Rotors are assembled at the center of the wings, and the main concept behind this design is by rotating the rotors it will benefit the drone in thrust generation. The tilt-wing system features a wing, which is horizontal for traditional forward flight and rotates upwards for vertical take-off and landing, unlike the tilt-rotor design that only the engine and propellers rotate. However, motor or wing tilting mechanism requires complex software and hardware like adding extra servos that will lead to an increase of weight and complexity. 


\section{Conceptual Design}

\subsection{Materials Selection}

Drones with a lighter structure result in an increase of efficiency, which means the flight will be able to cover larger areas. The amount reduced in structure weight can allow an increase of payload. In aerospace or aeronautics application, material with higher stiffness is required. Another important parameter is the strength-to-weight ratio. The material should be able to handle high load. CFRP has a high stiffness; however, it can fail upon elongation. Carbon fiber composites have high specific stiffness and strength, which make it the best for this type of application. Another type of material would be the fiberglass reinforced plastic. Table 1 shows the decision matrix for materials selection.

Table 1: Materials Decision Matrix

$0=$ Poor $\mid 1=$ Satisfactory $\mid 2=$ Reasonable $\mid 3=$ Very Good $\mid 4$ = Excellent

\begin{tabular}{|c|c|c|c|c|c|}
\hline 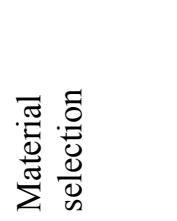 & 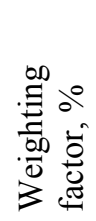 & 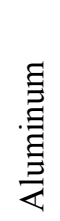 & 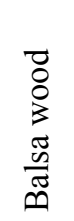 & 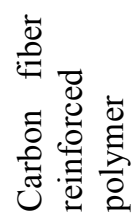 & 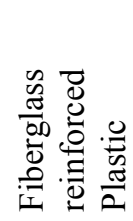 \\
\hline Cost & 10 & 1 & 4 & 1 & 3 \\
\hline Strength & 40 & 3 & 1 & 4 & 3 \\
\hline Weight & 30 & 1 & 3 & 4 & 2 \\
\hline Availability & 20 & 2 & 3 & 1 & 2 \\
\hline Total & 100 & 2 & 1.8 & 3 & 2.5 \\
\hline
\end{tabular}

\subsection{Wing Shape}

Based on the decision matrix in Table 2, the highest wing scoring is tapered, and the second highest is the rectangular wing. Both wings achieved highest required design parameters in terms of lift, drag, weight, stall resistance and stability. Tapered wing shape offers both enhanced efficiency and manufacturability. Taper resulted in lowering the induced drag due to larger span.

Table 2: Wing Shape Decision Matrix

$0=$ Poor $\mid 1=$ Satisfactory $\mid 2=$ Reasonable $\mid 3=$ Very Good $\mid 4=$ Excellent

\begin{tabular}{|c|c|c|c|c|c|}
\hline 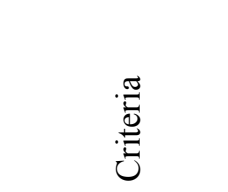 & 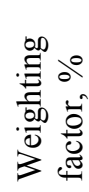 & 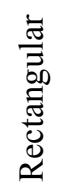 & 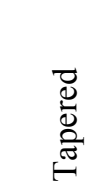 & 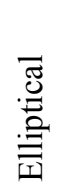 & $\begin{array}{l}\overrightarrow{0} \\
\overrightarrow{0} \\
\vec{b}\end{array}$ \\
\hline Lift & 30 & 3 & 4 & 2 & 2 \\
\hline Drag reduction & 30 & 3 & 4 & 4 & 2 \\
\hline $\begin{array}{l}\text { Ease of } \\
\text { manufacturing }\end{array}$ & 10 & 4 & 3 & 1 & 3 \\
\hline Stability & 15 & 3 & 4 & 2 & 2 \\
\hline Stall resistance & 15 & 3 & 3 & 2 & 2 \\
\hline Total & 100 & 3.1 & 3.75 & 2.5 & 2.1 \\
\hline
\end{tabular}

\subsection{Landing Gear}

Based on the results of the landing gear decision matrix (Table 3), the first choice will be Tricycle, followed by Tail-wheel and lastly Tandem. Tricycle (Fig. 3) is a type of landing gear consisting of a three-cycle, one wheel forward, which is called a nose wheel and two or more back wheels. The main rollers are typically attached to drone wing or fuselage structure. The numbers and positions can vary. The rollers enhance stability and ensure protection in the event of a one roller failure. During ground operations, the nose wheel enables the drone to change directions. The benefits of the drone design allow for faster braking without drone nosing, and for optimal placement of the center of gravity ground looping of drone is prevented.

\section{Table 3: Landing Gear Decision Matrix}

$0=$ Poor $\mid 1=$ Satisfactory $\mid 2=$ Reasonable $\mid 3=$ Very Good $\mid 4=$ Excellent

\begin{tabular}{|c|c|c|c|c|}
\hline 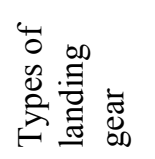 & 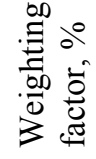 & 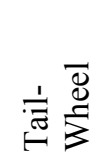 & 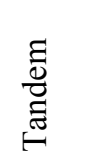 & 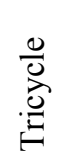 \\
\hline Weight & 10 & 3 & 2 & 3 \\
\hline Take-off & 25 & 3 & 2 & 4 \\
\hline $\begin{array}{l}\text { Ground } \\
\text { Handling }\end{array}$ & 25 & 4 & 1 & 3 \\
\hline Drag & 10 & 2.5 & 1.5 & 4 \\
\hline Durability & 15 & 3 & 2 & 4 \\
\hline $\begin{array}{l}\text { Propeller } \\
\text { Clearance }\end{array}$ & 15 & 4 & 1.5 & 3 \\
\hline Total & 100 & 3.35 & 1.625 & 3.50 \\
\hline
\end{tabular}




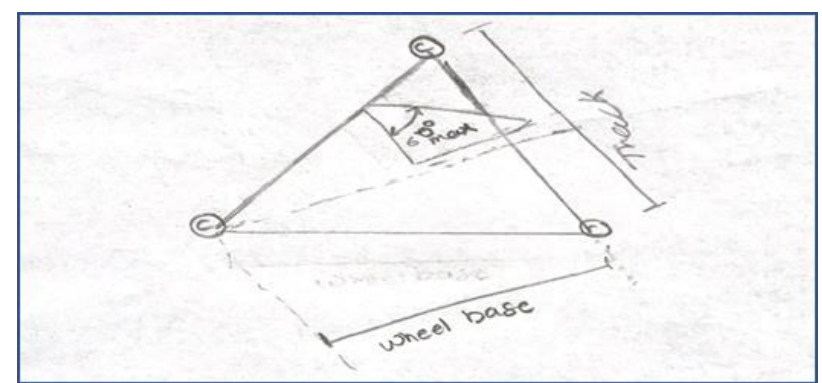

Figure 3: Tricycle sketch

\subsection{Airfoil Selection}

The following airfoils as shown in Fig. 4 were considered during the design process due to their respective advantages [9]. After conducting the XFLR5 aerodynamics analysis, the choice of airfoil is NACA 4412. The airfoil was chosen since it has the highest lift-to-drag ratio. This parameter has a high impact in increasing the drone aerodynamics efficiency, which will allow it to cover larger areas with minimum power.
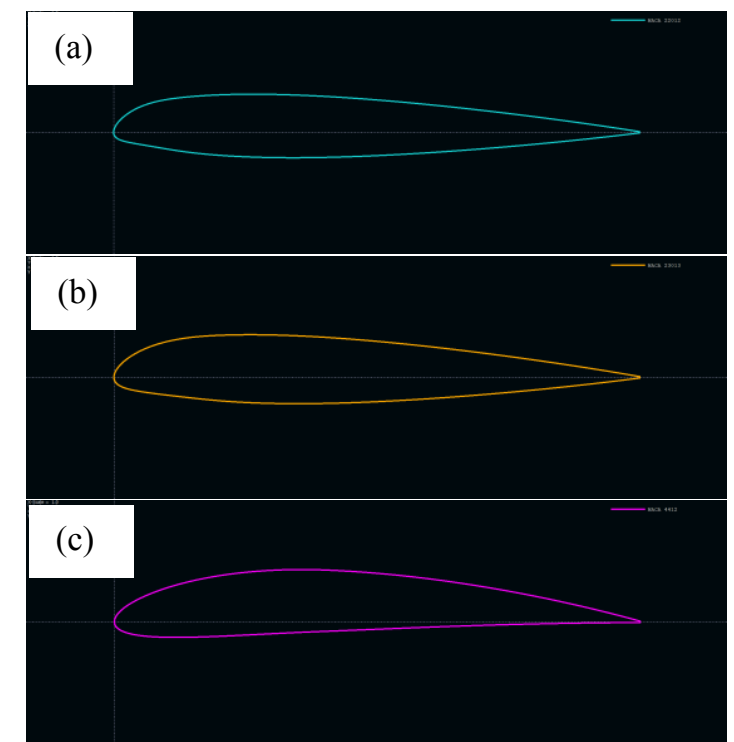

Figure 4: Airfoil types (a) NACA 2012 (b) NACA 23015 (c) NACA 4412

\subsection{Thermal Camera}

Thermal camera selection was based on the market research as per required mission. The goal was to find a lightweight, high-pixel thermal camera that can withstand certain degrees of vibrations due to flight dynamics. Weight reduction is an important parameter since it directly affects the design of the drone. Accuracy of the camera is significant to avoid misreading of human body temperatures. The camera installed in the hybrid drone was the Vue Pro R 336, $45^{\circ}$ radiometric drone thermal camera with a resolution of $640 \times 512$ pixels, as shown in Fig. 5 .

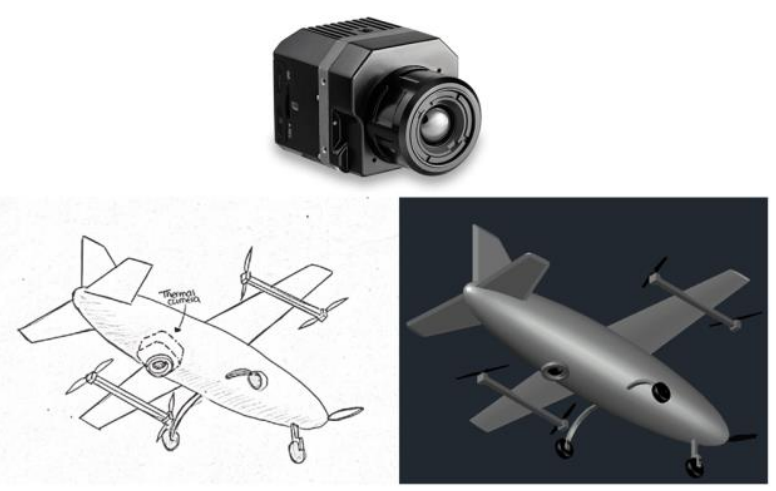

Figure 5: Thermal camera and its location

\section{Simulation Technique}

CFD solves the Navier-Stokes equation integrated with continuity equation. Equation 1 shows the steady state Navier-Stokes Equation.

$$
\rho(V \cdot \nabla V)=-\nabla p+\mu \nabla^{2} V+S
$$

where $\rho$ is the density, $V$ is resultant vector of velocity components, and $p$ is the pressure. The continuity equation is shown in Eq.2.

$$
(\nabla \cdot \rho V)=0
$$

The turbulence model chosen for this study is SST K-Omega (SST k- $\omega$ ) in ANSYS Fluent, which provides accurate results for flow separation and turbulence simulation especially when analyzing post-stall conditions. The standard equation for SST $\mathrm{k}-\omega$ was given in [11], shown as the following:

$$
\frac{\partial k}{\partial t}+U_{j} \frac{\partial k}{\partial x_{j}}=P_{k}-\beta^{*} k \omega+\frac{\partial}{\partial x_{j}}\left[\left(v+\sigma_{k} v_{T}\right) \frac{\partial k}{\partial x_{j}}\right]
$$

where $v_{T}$ is the kinematic eddy viscosity. The turbulence model was successfully used in the other vortex generator applications that further details can be found in [12-13]. The transport equations for the SST K-Omega (SST k- $\omega$ ) is provided as the following [14-15]:

$\frac{\partial}{\partial t}(\rho k)+\frac{\partial}{\partial x_{i}}\left(\rho k u_{i}\right)=\frac{\partial}{\partial x_{j}}\left[\left(\mu+\frac{\mu_{t}}{\sigma_{k}}\right) \frac{\partial k}{\partial x_{j}}\right]+\widetilde{G}_{k}-$
$Y_{k}+S_{k}$

Model verification is done by varying the mesh intensity. The edge size of the mesh is varied and the results obtained are compared (Table 4). It can be noticed that Mesh 2 and 3 gave almost the same values and Mesh 1 is a little deviated from the results of mesh 2 and 3. Figure 6 shows drone geometry meshing. 
Table 4: Mesh Intensity Results

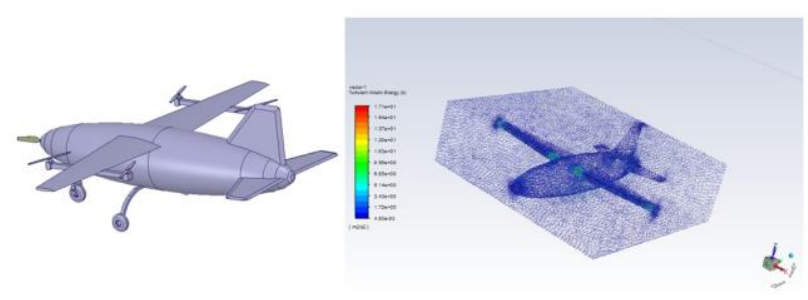

Figure 6: Geometry meshing

\section{Results and Discussions}

Firstly, the CFD runs were done at angle attack of $0^{\circ}$ (i.e. standard operation). The purpose is to observe the relation between increasing velocity and $L / D$. The results show that there is a slight increase in $L / D$ with a high increase of velocity. The reason of slight increase in $L / D$ with increase of velocity is because of the airfoil characteristics, in order for a wing to generate more lift the angle of attack must be increased; however, these the runs are done at a stationary angle of attack $0^{\circ}$. It shows that increasing velocity would cause a slight increase in lift, but it needs to come with an increase of angle of attack for the $L / D$ to rise. Referring to Table 5, the maximal value of lift is at angle of attack of $15^{\circ}$. Significant increase of drag is observed from angles of attack greater than $5^{\circ}$ and maximal lift to drag ratio is at angle of attack of around $10^{\circ}$.

Table 5: Lift and Drag Results at Various AoA

\begin{tabular}{|l|l|l|l|l|}
\hline $\begin{array}{l}\text { Velocity } \\
(\mathrm{m} / \mathrm{s})\end{array}$ & AoA & $\begin{array}{l}\text { Lift, } L \\
(\mathrm{~N})\end{array}$ & $\begin{array}{l}\text { Drag, } D \\
(\mathrm{~N})\end{array}$ & $L / D$ \\
\hline 14 & $0^{0}$ & 73.87 & 64.04 & 1.15 \\
\hline 14 & $5^{0}$ & 337.34 & 71.68 & 4.7 \\
\hline 14 & $10^{0}$ & 839.52 & 109.20 & 7.68 \\
\hline 14 & $15^{0}$ & 1110.96 & 173.68 & 6.39 \\
\hline
\end{tabular}

The subsequent CFD results show that the design operates best at the velocity of $20 \mathrm{~m} / \mathrm{s}$ and AoA of $10^{\circ}$. This demonstrates that the hybrid drone is able to operate at high-speed condition (about 70 $\mathrm{km} /$ hour) and various wind conditions. The velocity speed operation depends on the geometry and drag produced from the flight condition. It could be enhanced by smoothening the surface and avoiding sharp edges as well as turbulence enhancement methods [16-20].

Landing gear is playing the major components in the drone for take-off and landing safely to provide the required stability of the aircraft on the ground. Consequently, it should handle the load through the transmit parts. The safety factor is one of the major concerns in the optimization of the landing gears structure. It is measured for different

\begin{tabular}{|l|l|l|l|}
\hline Mesh Type & Mesh 1 & Mesh 2 & Mesh 3 \\
\hline Elements & 73,036 & 207,822 & 630,214 \\
\hline Lift Force (N) & 4.578 & 4.932 & 5.038 \\
\hline Drag Force (N) & 1.123 & 1.085 & 0.962 \\
\hline $\begin{array}{l}\text { Improvement in } \\
\text { L/D (\%) }\end{array}$ & 0.0 & 11.5 & 28.4 \\
\hline
\end{tabular}

loading conditions after a thorough analysis of stress along the structure. Optimizing the safety factor means preserving its value in a relatively safe range after weight optimization. In this research, the factor of safety using ANSYS structural analysis was around 15, and this is due to the type of material properties used with the rough environment conditions of flying, as shown in Fig. 7. The structural analysis shows that it was able to handle the required stresses in various flight conditions.

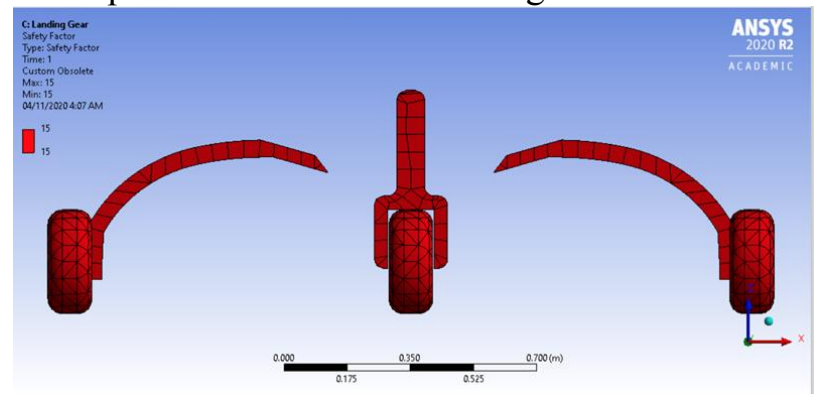

Figure 7: Landing gear safety factor

The framework has a significant effect on the endurance performance of solar aircraft. Analyzing solar aircraft can be concluded that the aerodynamic layout and design of the structure have a significant impact on the flight time and efficiency of the solar aircraft. Excessive force on the wing root causes material failure of the wing root beam, while excessive deformation of the wing causes aerodynamic performance degradation of the wing and damage to the battery, which affects aircraft endurance. There are several aerodynamic layout shapes suitable for solar UAV, such as traditional layout, flight wing layout, double strut layout, tandem wing layout, V-tail layout [21-22]. With total wingspan of $88 \mathrm{~cm}$, each wing can accommodate 5 solar cells (15.6 cm each length) producing a total of $5.5 \mathrm{~V}$ (Fig. 8). This provides sufficient power for long-range flying time of approximately 1.5 hours although careful consideration should be exercised due to the large wing deflection deformations during flight, which can cause severe aero-elastic problems. 

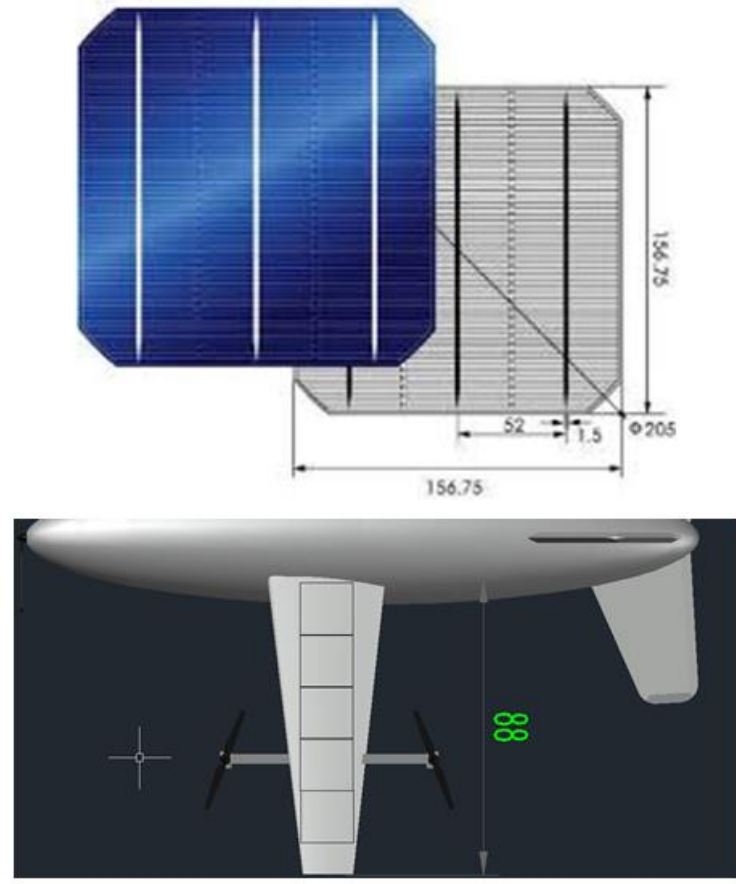

Figure 8: Solar panel position on wing

The following figure (Fig. 9) shows the final design of the hybrid drone whilst Fig. 10 illustrates the drone application sketch. Table 6 displays the drone specification sheet based on the analysis and calculations for the intended application. The drone can be further tested for reliability using the methods of [23-32].

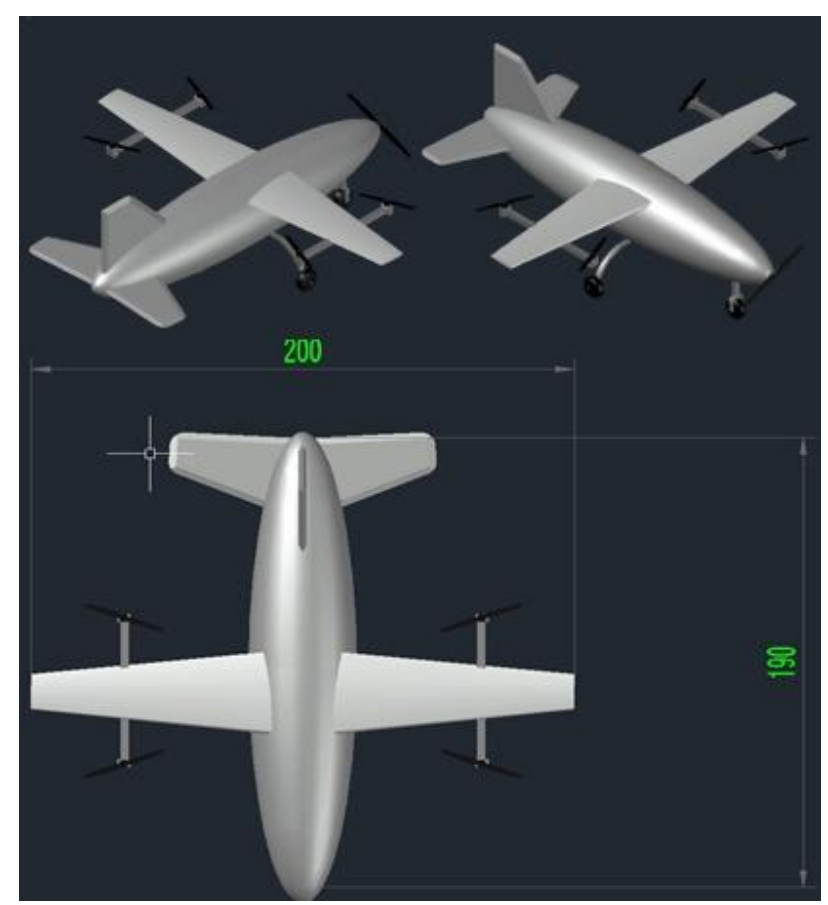

Figure 9: Hybrid drone final design

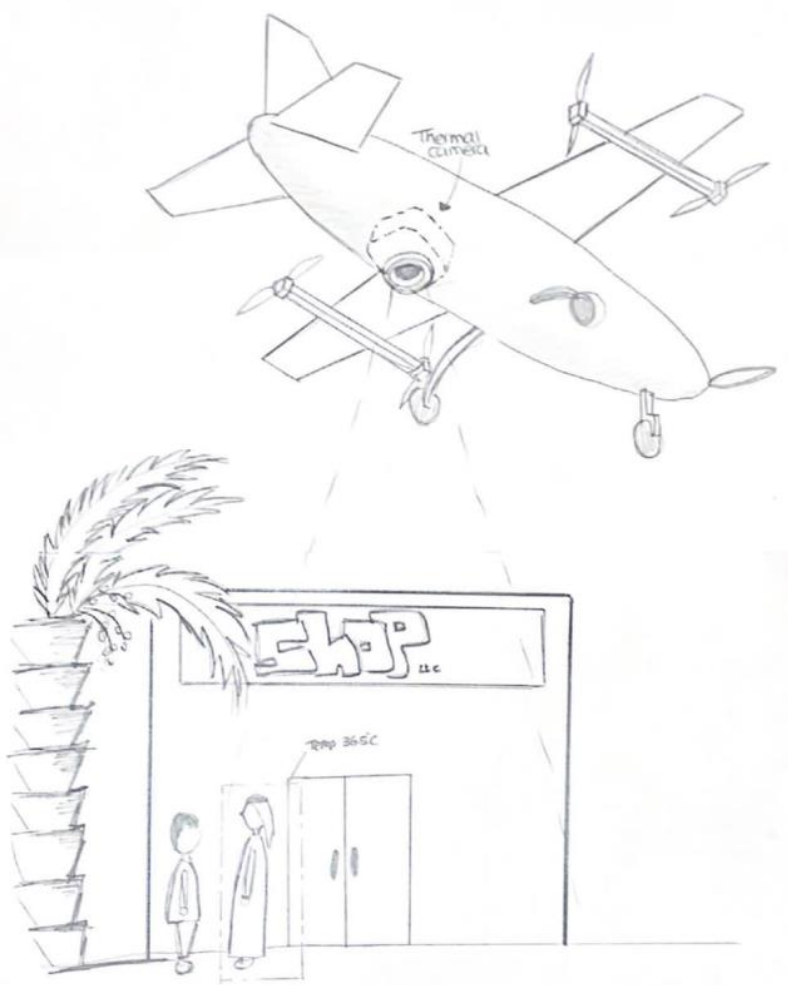

Figure 10: Drone application sketch Table 6: Hybrid Drone Specification Sheet

\begin{tabular}{|c|c|c|}
\hline \multicolumn{3}{|c|}{ Fixed wing and multi-copter combination drone } \\
\hline Quantity & Component & Weight, g \\
\hline 1 & (5.1)" propellers & 18 \\
\hline 1 & (7)" propeller & 9.1 \\
\hline 4 & $\begin{array}{l}\text { Batteries (Tattu 14.8V 75c 4S } \\
1050 \mathrm{mAh})\end{array}$ & 489.9 \\
\hline 1 & $\begin{array}{l}\text { Flir Vue Pros R thermal } \\
\text { camera }\end{array}$ & 92 \\
\hline 5 & $\begin{array}{l}\text { Motors (Bhobby 3B-R } 2207 \\
\text { Pro) }\end{array}$ & 147.5 \\
\hline 10 & Solar panel & 130 \\
\hline \multirow[t]{3}{*}{8} & Solar panel & $156 \mathrm{~mm}^{2}$ \\
\hline & $\begin{array}{l}\text { Flight duration (fixed wing } \\
\text { mode) }\end{array}$ & 85 mins \\
\hline & $\begin{array}{l}\text { Flight duration (Multi-copter } \\
\text { mode) }\end{array}$ & 22 mins \\
\hline
\end{tabular}

\section{Conclusion}

The drones' importance nowadays keep increasing due to the variety of application in many sectors, primarily for saving lives, rescuing the environment and empowering scientists through inventions. The hybrid drone solves a new challenge, which is human body temperature scanning while ensuring no human contact. This paper draws together the main concept behind this project, which is having a hybrid drone 
system that can measures the human body temperature for larger industrial areas roughly about $50 \mathrm{~km}^{2}$ without any human intervention during this pandemic COVID-19 period. To achieve this task, a hybrid solar-powered drone is introduced to take advantage of the high range fixed wing, which is able to fly for an approximate of 1.5 operational hours and maneuverable drone that will achieve stationary flying condition (i.e. no vibrations) to take human body temperatures. Maximum lift coefficient depends upon the airfoil shape, leading-edge slot or slat geometry, Reynolds number, flap geometry and span, surface texture, wing geometry and interference from other parts of the aircraft such as the fuselage, nacelles, or pylons. Therefore, with the technical advancements the hybrid drone was customized into different forms for vertical take-off with fixed wing and multi-rotatory propellers with the specification of altitude flying around 3-3.5 meters to cover a close area while scanning the human temperatures for larger areas. To decrease the weight and provide high altitude coverage, the drone is made of light composite material of carbon fiber. The future plan for the project includes design of collision avoidance system and flight automation.

\section{References}

[1] SP Yeong, LM King, SS Dol. "A Review on Marine Search and Rescue Operations Using Unmanned Aerial Vehicles". International Journal of Mechanical, Aerospace, Industrial, Mechatronic and Manufacturing Engineering, vol. 9, no. 2, pp. 396-399, 2015.

[2] Abid Abdul Azeez, Mohamed Gadala, Nasr Al Khudhiri, Sharul Sham Dol. "Aerodynamics Optimization of RC Plane Winglet." 2019 8th International Conference on Modeling Simulation and Applied Optimization (ICMSAO), pp. 1-5. IEEE, 2019.

[3] Abdul Qader Hasan, Nasr M. Al-Khudhiri, Muhammad A. Iqbal, Sharul Sham Dol, Abid Abdul Azeez, Mohamed S. Gadala, "Aerodynamics Analysis on Wings with Winglets and Vortex Generators", WSEAS TRANSACTIONS ON FLUID MECHANICS, 15, pp. 193-201, 2020.

[4] Sharul Sham Dol. "Aerodynamic optimization of unmanned aerial vehicle for offshore search and rescue (SAR) operation." IOP Conference Series: Materials Science and Engineering, vol. 715, no. 1, p. 012015. IOP Publishing, 2020.
[5] Muhammad Ahsan Iqbal, Abdul Qader Abdullah, Sharul Sham Dol. "Design of selfpowered surveillance RC aircraft." International Journal of Engineering Research in Mechanical and Civil Engineering (IJERMCE) Volume 5, February 2020.

[6] Eid, Saif Eldin, and Sharul Sham Dol. "Design and Development of Lightweight-High Endurance Unmanned Aerial Vehicle for Offshore Search and Rescue Operation." In 2019 Advances in Science and Engineering Technology International Conferences (ASET), pp. 1-5. IEEE, 2019.

[7] A. F. El Ghazali and S. S. Dol. "Aerodynamic Optimization of Unmanned Aerial Vehicle through Propeller Improvements." Journal of Applied Fluid Mechanics, vol. 13, no. 3, pp. 793-803, 2020.

[8] A. Q. Abdullah and S. S. Dol, "Aerodynamic Investigation and Design of Dimpled-Surface Airfoil for UAV Propellers," 2020 Advances in Science and Engineering Technology International Conferences (ASET), Dubai, United Arab Emirates, 2020, pp. 1-5.

[9] Siew Ping Yeong and Sharul Sham Dol. "Aerodynamic Optimization of Micro Aerial Vehicle". Journal of Applied Fluid Mechanics, vol. 9, no. 5, pp. 2111-2121, 2016.

[10] Chamata, J. "A Proposal for the Adoption of Unmanned Aerial Technology in Malaysia." In 4th Borneo Research Education Conference, BREC. 2016.

[11] ABDULLAH, Abdul Qader, Abid Abdul Azeez, Sharul Sham Dol, Mohammad Khan, and Mior Azman Meor Said. 2020. "SIMULATION STUDY ON VORTEXINDUCED VIBRATION AIR WAKE ENERGY FOR AIRPORT RUNAWAY APPLICATION: A PRELIMINARY ANALYSIS." Platform : A Journal of Engineering, [S.1.], v. 4, n. 3, 38-47.

[12] Dol, Sharul Sham, Siaw Khur Wee, Hiang Bin Chan, Perumal Kumar. 2019. "Turbulence Characteristics behind a Flexible Vortex Generator" WSEAS TRANSACTIONS ON FLUID MECHANICS, 14, 1-7.

[13] Dol, Sharul Sham, Hiang Bin Chan, Siaw Khur Wee, Kumar Perumal. 2020. "The effects of 
flexible vortex generator on the wake structures for improving turbulence." IOP Conference Series: Materials Science and Engineering, vol. 715, no. 1, p. 012070. IOP Publishing.

[14] O. M. Kassem, A. Q. Abdullah, S. S. Dol, M. S. Gadala and M. S. Aris, "CFD Analysis on the Single-Phase Flow Electrical Submersible Pump Performance Curve," Platform: A Journal of Engineering, vol. 4, no. 4, 5 January 2021.

[15] Hiang Bin Chan, Tshun Howe Yong, Perumal Kumar, Siaw Khur Wee, Sharul Sham Dol. "The Numerical Investigation on the Effects of Aspect Ratio and Cross-Sectional Shape on the Wake Structure behind a Cantilever". ARPN Journal of Engineering and Applied Sciences, vol. 11, no. 16, pp. 9922-9932, 2016.

[16] M. O. Khan, M. A. Khan and S. S. Dol, "Effects of Chevrons on the Acoustic Noise and Velocity Patterns of Aircraft Nozzles," 2020 International Conference on Decision Aid Sciences and Application (DASA), Sakheer, Bahrain, 2020, pp. 845-849.

[17] DOL, Sharul Sham; CHAN, Hiang Bin; WEE, Siaw Khur. FSI SIMULATION OF A FLEXIBLE VORTEX GENERATOR AND THE EFFECTS OF VORTICES TO THE HEAT TRANSFER PROCESS. Platform : A Journal of Engineering, [S.1.], v. 4, n. 2, p. 5869, June 2020.

[18] Sharul Sham Dol, Chan Hiang Bin. "FluidStructural Interaction Simulation of Vortices behind a Flexible Vortex Generator." 2019 8th International Conference on Modeling Simulation and Applied Optimization (ICMSAO), pp. 1-5. IEEE, 2019.

[19] SF Wong, SS Dol. "Simulation Study on Vehicle Drag Reduction by Surface Dimples". International Journal of Mechanical, Aerospace, Industrial, Mechatronic and Manufacturing Engineering, vol. 10, no. 3, pp. 560-565, 2016.

[20] SS Dol. "Design and Development of a Prototype Vehicle for Shell Eco-Marathon". International Journal of Mechanical, Aerospace, Industrial, Mechatronic and Manufacturing Engineering, vol. 10, no. 3, pp. 546-552, 2016.
[21] S. S. Pervaiz, A. Abdullah and S. S. Bin Dol, "Simulation Study of a Solar Glider Design," 2020 International Conference on Decision Aid Sciences and Application (DASA), Sakheer, Bahrain, 2020, pp. 355-359.

[22] Sh. Sham Dol; S. Shahid Pervaiz; M. Uzair; Sh. Khalid Bashir; M. Mustafa Elzughbi. "Design of Solar-powered Endurance Glider with Vortex Generators". Journal of Renewable Energy Research and Application (RERA), 2020.

[23] TH Yong, SS Dol. "Design and Development of Low-Cost Wind Tunnel for Educational Purpose". IOP Conference Series: Materials Science and Engineering, 78, 012039, 2015.

[24] M Zahari, SS Dol. "Design and Development of Low-Cost Water Tunnel for Educational Purpose". IOP Conference Series: Materials Science and Engineering 78, 012040, 2015.

[25] Dol, Sharul Sham. "An Improved Design of Low-Cost Wind Tunnel for Educational Purpose in Fluid Dynamics." 10th Int'l Conference on Advances in Engineering, Science, Technology \& Sustainable Development (ESTSD-18) July 25-27, 2018 Bali (Indonesia).

[26] Sharul Sham Dol, Mohd Arief Mohd Nor and Muhamad Khairun Kamaruzaman. "An improved smoke-wire flow visualization technique". Proceedings of the 4th WSEAS International Conference on Fluid Mechanics and Aerodynamics, Elounda, Greece, August 21-23, pp. 231-236, 2006.

[27] Sharul Sham Dol, Mohd Arief Mohd Nor and Muhamad Khairun Kamaruzaman. "Flow visualization of the vortex shedding of a stationary circular cylinder by an improved smoke-wire technique". WSEAS Transactions on Fluid Mechanics, Issue 6, Volume 1, pp.745-752, 2006.

[28] Sharul S. Dol, U. Azimov and Robert J. Martinuzzi. "Statistical description in the turbulent near wake of rotating circular cylinder". International Journal of Mechanical, Aerospace, Industrial, Mechatronic and Manufacturing Engineering, vol. 6, no. 12, pp. 2706-2710, 2012. 
[29] Sharul S. Dol. "Weakened Vortex Shedding from a Rotating Cylinder." International Journal of Mechanical and Mechatronics Engineering, vol. 7, no. 10, pp. 2013-2020, 2013.

[30] Sharul Sham Dol. "Proper orthogonal decomposition analysis of vortex shedding behind a rotating circular cylinder". EPJ Web of Conferences, vol. 114, 02019, 2016.

[31] Dol, S. S., and R. J. Martinuzzi. "Patterns of vortex shedding from a rotating cylinder." In Proceedings of 7th CUTSE Conference, Curtin University, Sarawak, vol. 6, no. 12. 2012.

[32] Zahari, M., H. B. Chan, T. H. Yong, and S. S. Dol. "The effects of spring stiffness on vortexinduced vibration for energy generation." In IOP Conference Series: Materials Science and Engineering, vol. 78, no. 1, p. 012041. IOP Publishing, 2015.

[33] Mior A. Said, D Bong, M. S. Mohd and S. S. Dol. "Prototype Development of Thermoelectric Generator System for Exhaust Heat Recovery of an Automobile." ARPN Journal of Engineering and Applied Sciences, vol. 11, no. 20, pp. 12217-12221, 2016.

\section{Creative Commons Attribution License 4.0 (Attribution 4.0 International, CC BY 4.0)}

This article is published under the terms of the Creative Commons Attribution License 4.0

https://creativecommons.org/licenses/by/4.0/deed.en US 